ARTÍCULOS ORIGINALES Rev Chil Salud Pública 2016; Vol 20 (2): 113-121

\section{APRENDIZAJE BASADO EN PROBLEMAS COMO RECURSO PEDAGÓGICO EN EL CAMBIO DE LA CONDUCTA SEDENTARIA EN JÓVENES UNIVERSITARIOS}

Problem- based learning as a PeDagogic resource to change

THE SEDENTARY BEHAVIOR AMONG COLLEGE YOUTH

\title{
RESUMEN
}

Introducción: El sedentarismo es considerado un problema de salud pública por sus implicaciones para la salud física y emocional afectando a entre un 85 y un $90 \%$ de los jóvenes universitarios.

Objetivo: Conocer el efecto de la implementación de la metodología del Aprendizaje Basado en Problemas (ABP) como recurso pedagógico en el cambio de la conducta sedentaria en jóvenes universitarios del curso Actividad Deportiva de la Universidad de Costa Rica.

Material y Métodos: Participaron 96 universitarios (48 hombres y $48 \mathrm{mu}$ -

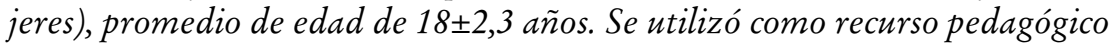
la metodología del ABP para propiciar el cambio de la conducta sedentaria. El cambio de la conducta sedentaria se evaluó por medio del aumento de la actividad física (AF) realizada durante la semana. Se realizó el Pre-test, para conocer la cantidad de días a la semana que los universitarios dedicaban a la práctica de AF. Se implementó el método ABP durante 12 semanas, posteriormente se realizó el Post-test, para conocer la cantidad de AF que realizaron durante la semana posterior a la implementación del recurso pedagógico. Se aplicó un ANOVA de dos vías con el fin de identificar diferencia significativa entre mediciones.

Resultados: Se identificaron cambios significativos en la cantidad de AF entre la medición Pre-test y Post-test; sin embargo, no se identificó diferencia significativa en las mediciones obtenidas entre hombres y mujeres.

Conclusión: La implementación de la estrategia de enseñanza ABP permitió un cambio en la conducta sedentaria en un grupo de jóvenes universitarios, propiciando un aumento significativo en la práctica de AF realizada durante la semana. Se identificó que la estrategia del ABP tuvo el mismo efecto positivo en los hombres como en las mujeres.

Palabras clave: Actividad física, Aprendizaje Basado en Problemas, ejercicio, jóvenes universitarios, sedentarismo.

Orlando Maroto

Facultad de Odontología Escuela de Formación Docente

Universidad de Costa Rica

Trabajo recibido el 15 de enero 2016. Aceptado el 20 de octubre 2016. 


\section{ABSTRACT}

Introduction: Sedentary behavior is considered to be a public health problem due to its effect on people's physical and emotional health. It affects between $85 \%$ and $90 \%$ of college youth.

Objective: To implement the Problem-Based Learning (PBL) methodology as a pedagogic resource to change the sedentary behavior among college youth.

Methods and Material: A total of 96 college youth participated (48 males and 48 females), average age $18 \pm 2.3$ years. The pedagogic PBL methodology was the resource used to promote a change in sedentary behavior. This was screened through the increase of physical activity (PA) performed during the week. Students had a pre-evaluation that determined the amount of days in the week that they were doing PA. The PBL method was implemented for 12 weeks; afterwards, a post-evaluation determined the amount of $P A$ that they had performed during the week following the implementation of the pedagogic resource. A two-lined ANOVA was applied so that the significant difference between measurements could be identified.

Results: There were significant changes in the amount of PA between the pre-test and the post-test measurements; however, no significant differences between measurements obtained among men and women were identified.

Conclusion: The implementation of the PBL strategy allowed a change in the sedentary behavior among a group of college youth. It promoted a significant increase in the performance of $P A$ done during a week. The PBL strategy had the same positive effect in both men and women.

Keywords: Physical activity, Problem Based Learning, Exercise, College youth, Sedentary behavior.

\section{INTRODUCCIÓN}

El sedentarismo es considerado un problema de salud pública debido a sus graves implicaciones tanto para la salud física como emocional. $^{1-5}$

La Organización Mundial de la Salud $(\mathrm{OMS})^{6}$ considera el sedentarismo al igual que la obesidad como uno de los problemas más importantes de salud pública, por su alta prevalencia y sus consecuencias sobre la expectativa de vida; razones por las cuales se ha motivado a los gobiernos a tratar de combatir el sedentarismo desde diversos ambientes, ${ }^{7}$ ya que los costos económicos que esta problemática reporta anualmente son significativos y podrían redirigirse hacia otros campos de inversión. ${ }^{8}$

A nivel físico una persona sedentaria tiene un mayor riesgo de padecer múltiples enfermedades crónicas no transmisibles (ECNT), como lo son la diabetes mellitus, la hipertensión, enfermedades cardiovasculares, entre otras; afectando a la mayoría de la población mundial y provocando un aumento en los ín- dices de mortalidad. Y a nivel emocional, se ha descubierto que una persona sedentaria tiene mayor probabilidad de padecer insomnio, depresión, ansiedad, estrés, entre otros. 1 Se calcula que entre el 85 y el $90 \%$ de los jóvenes estudiantes universitarios de todo el mundo son sedentarios 8 y más de dos millones de muertes anuales en la población adulta se le atribuyen. ${ }^{9}$

Las universidades, como ambientes de formación integral de individuos, deben ser de las principales instituciones de un país en propiciar por medio del conocimiento conductas más apropiadas para el desarrollo integral del ser humano, entre ellas una actitud positiva hacia la práctica de la actividad física, con el fin de disminuir el sedentarismo en sus estudiantes. Por lo tanto éste requiere de un modelo de enseñanza que le brinde la capacidad de un autoaprendizaje y le permita propiciar cambios en sus conductas a lo largo de la vida.

El Aprendizaje Basado en Problemas (ABP), también conocido como PBL por sus siglas en inglés (Problem-Based Learning), ${ }^{10}$ 
empleado comúnmente en las Ciencias de la Salud ${ }^{11-15}$ y extendido a otras disciplinas como Administración, Ciencias Sociales, Derecho, Economía, entre otras, se ha presentado desde sus inicios como un recurso pedagógico inductivo, innovador, que se caracteriza por estar el aprendizaje centrado en el estudiante. ${ }^{16}$

La metodología del ABP se desarrolló en primer lugar en los años sesenta en la Facultad de Medicina de la Universidad de McMaster, Canadá, y en la escuela de Medicina de la Universidad de Case Western Reserve, Estados Unidos ${ }^{10,17}$ y ha sido aplicada desde 1968 en América y desde 1974 en Europa. ${ }^{18}$

El ABP se define como un método de aprendizaje basado en el principio de usar problemas reales como punto de partida para la adquisición e integración de nuevos conocimientos, valores, habilidades y actitudes ${ }^{19}$ que, a su vez, facilitan cambios de conductas.

La enseñanza basada en ABP se fundamenta en el contexto del constructivismo, mediante el cual el estudiante busca y selecciona la información, razona e integra los conocimientos previos y adquiridos, dando finalmente unas posibilidades diagnósticas o terapéuticas a un problema planteado. ${ }^{11} \mathrm{Sin}$ embargo, el problema debe ser retador, interesante y motivador para que el alumno se interese por buscar la solución. ${ }^{20-22}$

Entre las principales características del método ABP están: 1. trabajo en grupos pequeños; 2. el papel del docente se limita a ser un facilitador o guía en el proceso de análisis del problema y en el funcionamiento del grupo; 3. el problema es un vehículo para el desarrollo de habilidades de resolución de problemas; 4. los estudiantes analizan el problema y seguidamente determinan las necesidades de aprendizaje. ${ }^{21}$

Las universidades no solamente deben transmitir o enseñar información disciplinar propiamente dicha, sino que deben enseñar procedimientos, formas de utilizar el conocimiento por medio de estrategias pedagógicas que faciliten progresivamente la disminución del sedentarismo y fomenten la continuidad en la práctica de la actividad física.
El propósito de la presente investigación es conocer el efecto que tiene sobre la adquisición de una vida activa en un curso universitario ofertado como actividad deportiva en la Universidad de Costa Rica, la implementación de la metodología del Aprendizaje Basado en Problemas (ABP) como recurso pedagógico en el cambio de la conducta sedentaria en jóvenes universitarios.

\section{METODOLOGÍA}

\section{Tipo de estudio}

Investigación de tipo cuasi experimental, donde la intervención no aleatorizada se aplica a un determinado grupo y se estiman las diferencias antes y después.

\section{Variable de estudio}

Conducta sedentaria: nivel de actividad física que no supera los 30 minutos de ejercicio continuo al día, realizado al menos tres veces a la semana. ${ }^{23}$ El cambio de la conducta sedentaria se evaluó por medio del aumento de la cantidad de días a la semana que los universitarios reportaron por medio de un test autoadministrado, el cual se aplicó al inicio (diagnóstico Pre-test) y al final del estudio (Post-test).

\section{Estudiantes participantes}

En el estudio participaron 96 jóvenes (48 hombres y 48 mujeres), estudiantes universitarios pertenecientes a la Universidad de Costa Rica, con edades comprendidas entre los 17 y los 25 años (18 $\pm 2,34$ años).

\section{Recurso pedagógico}

Para efecto del estudio se utilizó como recurso pedagógico la estrategia de enseñanza del Aprendizaje Basado en Problemas (ABP). ${ }^{10}$

Se implementó como base el modelo de ABP $4 \times 4$ diseñado por Alfredo Prieto. ${ }^{24}$ Básicamente, el modelo se desarrolló en cuatro escenarios de trabajo (trabajo con clase completa, trabajo en pequeño grupo con y sin tutor y 
trabajo individual), fundamentándose en que el estudiante busca, selecciona información, razona e integra los conocimientos previos y adquiridos para generar las posibilidades terapéuticas al problema planteado.

\section{Situación por resolver}

Por medio del método ABP se planteó para efecto del estudio el problema: “¿Cómo propiciar un cambio en la conducta sedentaria en los estudiantes del curso de Defensa Personal, de la Universidad de Costa Rica en el primer ciclo del 2015?”, problema que corresponde a la situación por resolver. El método permite que el estudiante con la guía del docente, el trabajo con otros estudiantes y con la práctica de las actividades propuestas, trabaje y lleve a cabo los pasos o etapas de forma sistemática para la solución del problema o situación por resolver.

\section{Escenario de trabajo}

El escenario de trabajo correspondió al curso de Defensa Personal, el cual pertenece a la Cátedra de Actividad Deportiva de la Escuela de Educación Física y Deporte de la Universidad de Costa Rica, curso que se imparte dos horas a la semana, durante 16 semanas.

\section{Procedimiento}

\section{Fases}

1. Pre-test diagnóstico. Al iniciar el curso se llevó a cabo el diagnóstico (Pre-test), por medio de un test autoadministrado, para conocer la cantidad de días a la semana que los estudiantes dedicaban a la práctica de actividades físicas o ejercicio, además de las razones por las cuales consideraban que lo hacían o no.

2. Lecciones con metodología $A B P$. Para lograr el cambio de la conducta sedentaria de los universitarios (situación por resolver), el docente implementó durante todas las lecciones el recurso pedagógico (método ABP). Los universitarios debían asistir $\mathrm{y}$ participar de todas las lecciones del curso; además se comprometían a seguir generando respuesta al problema planteado durante su tiempo libre.

3. Post-test. La implementación del método ABP se llevó a cabo durante 12 lecciones. Una vez finalizadas se volvió a aplicar el test autoadministrado (Post-test), para conocer la cantidad de días a la semana que realizaron actividad física posterior a las lecciones con la implementación de la metodología ABP, así como las razones que los motivaron a aumentarla o no.

\section{Análisis estadístico}

La estadística descriptiva fue calculada para los datos recolectados expresados como promedios \pm desviaciones estándar (DS). Se aplicó un análisis de varianza de dos vías para medidas repetidas (ANOVA), grupos (dos niveles) por mediciones (dos niveles); con el fin de identificar diferencia significativa entre mediciones utilizando un nivel de significancia estadística de alfa 0,05. El análisis estadístico se realizó con ayuda del software estadístico MedCalc versión 11.5.1 (Mariakerke, Bélgica).

\section{RESULTADOS}

La Tabla 1 presenta la estadística descriptiva para la edad de los sujetos. En la Tabla 2 se presenta la estadística descriptiva obtenida en el Pre-test y Post-test correspondiente a la cantidad de días a la semana en que cada grupo de universitarios realizó actividad física. La Tabla 3 presenta los principales factores que manifestaron los estudiantes por los cuales no realizaban actividad física (Pre-test), así como aquellos factores que motivaron el aumento de la actividad física o ejercicio durante la semana (Post-test).

El ANOVA 2x2 (grupos por mediciones), mostró cambios significativos en la medición Post-test con respecto al Pre-test $(\mathrm{p}<0.05)$; sin embargo, no se identificó diferencia significativa $(p>0.05)$ en las mediciones obtenidas entre hombres y mujeres. 
Tabla 1. Estadística descriptiva correspondiente a la edad de los estudiantes universitarios

\begin{tabular}{llllll}
\hline Edad (años) & Promedio & DS & $95 \%$ CI & Mínimo & Máximo \\
\hline Hombre & 19,5 & 3,0 & $18,64-20,39$ & 17,0 & 25,0 \\
Mujeres & 18,0 & 0,9 & $17,72-18,27$ & 17,0 & 22,0 \\
\hline
\end{tabular}

Tabla2. Estadística descriptiva correspondiente al pre-test y post-test de la cantidad de días a la semana en que cada grupo de universitarios realizó actividad física

\begin{tabular}{lllllll}
\hline $\begin{array}{l}\text { Actividad Física } \\
\text { (Días a la semana) }\end{array}$ & Promedio & DS & $\mathbf{9 5 \% ~ C I}$ & Mínimo & Máximo & p \\
\hline Pre-test & & & & & & \\
Hombres & 1,5 & 1,5 & $1,08-2,00$ & 0,0 & 2,0 & \\
Mujeres & 1,5 & 1,7 & $1,02-2,01$ & 0,0 & 3,0 & \\
Post-test & & & & & & \\
Hombres & 2,6 & 1,4 & $2,22-3,06$ & 1,0 & 6,0 & $0,0001^{*}$ \\
Mujeres & 2,1 & 1,4 & $1,70-2,54$ & 0,0 & 7,0 & $0,0003^{*}$ \\
\hline "p $<0.05$ & & & & & &
\end{tabular}

Tabla 3. Factores que propiciaron la inactividad física (pre-test) o el aumento de la misma durante la semana (post-test) en jóvenes universitarios

\begin{tabular}{ll}
\hline Factores que propiciaron la inactividad física & $\begin{array}{l}\text { Factores que motivaron el aumento de la actividad } \\
\text { física }\end{array}$ \\
\hline $\begin{array}{l}\text { Falta de motivación hacia la práctica de la actividad fí- } \\
\text { sica o ejercicio. }\end{array}$ & $\begin{array}{l}\text { Reconocer los factores de riesgo que puede provocar el } \\
\text { sedentarismo en el ser humano. }\end{array}$ \\
$\begin{array}{l}\text { Falta de tiempo para realizar actividad física debido a las } \\
\text { actividades universitarias. }\end{array}$ & $\begin{array}{l}\text { Reorganización del uso del tiempo libre. } \\
\text { Implementar actividades positivas para la salud. }\end{array}$ \\
$\begin{array}{l}\text { Falta de tiempo para realizar actividad física debido a las } \\
\text { actividades universitarias y laborales. }\end{array}$ & $\begin{array}{l}\text { Reorganización del uso del tiempo libre. } \\
\text { Implementar actividades positivas para la salud. }\end{array}$ \\
$\begin{array}{l}\text { La actividad física es solo un mecanismo ambiental que } \\
\text { favorece el metabolismo energético, especialmente para } \\
\text { el control de peso. }\end{array}$ & $\begin{array}{l}\text { Reconocer los beneficios que propicia la actividad física, } \\
\text { no solo en el control de peso sino a nivel cognitivo, salud } \\
\text { mental, salud física y a nivel psicosocial. }\end{array}$ \\
\hline
\end{tabular}

En el Gráfico 1 se aprecia el cambio significativo de Pre-test a Post-test en los grupos de universitarios.

\section{DISCUSIÓN}

Los resultados del presente estudio mostraron que los universitarios al inicio del estudio (Pre-test) no realizaban la mínima cantidad de actividad física recomendada a la semana, identificando que el estilo de vida era sedentario. Las principales razones que expresaron estaban más relacionadas con los estilos de vida individuales que con factores externos, lo cual también se identificó en el estudio de López et al., ${ }^{25}$ tendencia actual informada por este mismo tipo de población. ${ }^{1}$

Con posterioridad a la implementación del método ABP durante las lecciones del curso para propiciar el cambio en la conducta sedentaria, se identificó un cambio significativo en la cantidad de días a la semana que los universitarios reportaron realizar actividad física o ejercicio. 
Gráfico 1: Porcentaje de universitarios que manifestaron que el método ABP propició el cambio en la conducta sedentaria, facilitando el aumento de la actividad física realizada durante la semana.

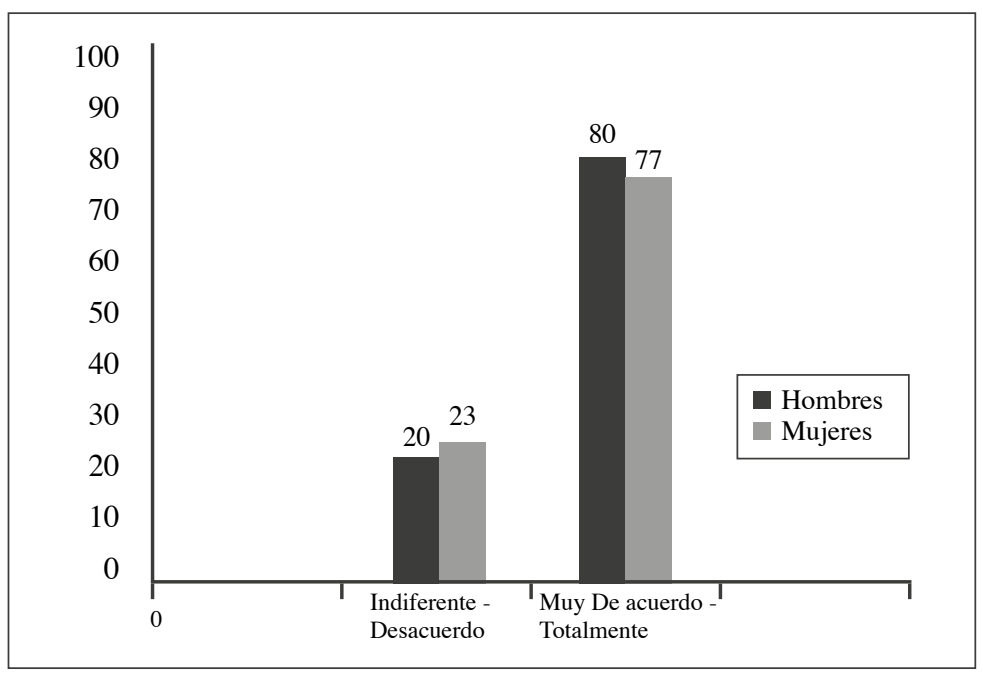

Con respecto al nivel de actividad física, se ha identificado que hay un mayor número de mujeres sedentarias que de hombres, ${ }^{26}$ aun cuando hasta la fecha no se tienen claras las razones de dicha diferencia por sexos. ${ }^{27-29} \mathrm{Sin}$ embargo, en el presente estudio no se presentó tal diferencia ya que se identificó que el método ABP generó el mismo efecto positivo tanto en hombres como en mujeres, lo cual se comprobó al no encontrarse diferencia significativa entre los resultados de ambos grupos de universitarios.

Se ha considerado que las posibles diferencias de actividad física tengan relación con los roles de género. Tradicionalmente en la cultura occidental el ejercicio y los deportes (en especial a nivel competitivo) han estado vinculados con los hombres como parte de la demostración de fuerza, resistencia y poderío. Para el caso de las mujeres, el rol tradicional en el desarrollo de actividad física ha estado asociado con las tareas del hogar; sin embargo, hoy en día las jóvenes universitarias tienen una responsabilidad social distinta que la de ser amas de casa, ${ }^{1}$ lo que pudo ser el factor por el cual se identificara el mismo nivel de actividad física que en los hombres.

Con el nivel moderado de actividad física registrado por los universitarios, se podrían prevenir las consecuencias del sedentarismo y propiciar una mejor calidad de vida. ${ }^{30}$ Entre los posibles beneficios que entrega un nivel moderado de actividad física están: 1 . a nivel del sistema nervioso, el aumento de la plasticidad neuronal, la elevación del nivel del factor neurotrófico derivado del cerebro, lo que favorece la memoria visual y el aprendizaje, ${ }^{31}$ la activación del sistema nervioso simpático y la liberación de noradrenalina y dopamina que ayudan a mejorar el estado de ánimo; ${ }^{1}$ 2. a nivel mental y psicológico, en la disminución de la ansiedad y depresión, aumento de la autoestima; $;^{32}$ 3. a nivel psicosocial, el fortalecimiento de la confianza en sí mismos, sentimientos de éxito, favorece las relaciones y la integración social, ${ }^{25} 4$. a nivel académico, en el desempeño escolar, sobre todo en lo que respecta a las matemáticas y la lectura. ${ }^{30-34}$

Este incremento en la cantidad de actividad física durante la semana dio por resultado que el método ABP permitiera por medio de la experiencia educativa, que el universitario cambiara la percepción sobre la necesidad de hacer actividad física varias veces a la semana; esto gracias a la implementación de los conocimientos teórico-prácticos, así como de aquellas experiencias previas en el proceso pedagógico y de investigación sobre el problema a resolver, además de facilitar una adecuada interacción docente-estudiantes. ${ }^{35}$ 
Otro de los factores que pudo propiciar el aumento en la actividad física semanal fue el protagonismo de los universitarios en el proceso de enseñanza-aprendizaje, como lo afirma Gómez et al., ${ }^{36}$ logrando que se concientizaran sobre la necesidad del ejercicio físico para su salud.

Se puede considerar que el efecto positivo que se obtuvo en el estudio fue producto, como lo señalan Molina et al., ${ }^{37}$ de que la implementación del método ABP en cualquier proceso de enseñanza-aprendizaje favorece a los participantes a adquirir conocimientos, identificar habilidades y desarrollar destrezas que les permitan brindar respuesta al problema planteado. Para efecto del estudio, lo que propició el método fue que los universitarios, al identificar el sedentarismo como aquella condición personal que puede generar una serie de alteraciones a nivel orgánico, y reflexionar sobre cómo resolver el problema planteado, lograron que los conocimientos teóricos adquiridos sobre los beneficios de la actividad física no solo quedaran como una reflexión de curso o un discurso filosófico, sino que lograron implementar en su tiempo libre las actividades física ejecutadas en el curso, como recurso en la adquisición y mantenimiento de aquellos aspectos relacionados con la salud.

Otro aspecto que pudo propiciar el efecto que tuvo el método ABP en el cambio de actitud hacia la práctica de la actividad física, fue que la adecuada utilización de la estrategia motiva a los universitarios a tener una mayor disposición y apertura en la búsqueda de soluciones a un problema planteado. ${ }^{37}$

Con respecto a las razones que brindaron los universitarios por las cuales aumentaron el nivel de actividad física durante la semana, éstas también fueron identificadas en otros estudios. ${ }^{1,25,27}$

En cuanto al interés que mostraron los universitarios hacia la actividad física propiciando el aumento de su práctica durante la semana, la mayoría de ellos estuvo de acuerdo en que fue el resultado de la implementación del método utilizado. Aunque en el estudio de Gómez et al. ${ }^{36}$ se evaluó una condición académica, los universitarios también estuvie- ron de acuerdo en que los logros obtenidos fueron el resultado de la implementación en el curso del método ABP.

Los resultados obtenidos permiten generar un espacio de reflexión en donde la universidad como institución educativa promotora del desarrollo de los procesos de enseñanza-aprendizaje, producción de conocimiento, acción social e investigación contemple -frente a una problemática silenciosa que está afectando a la población estudiantil- las necesidades de ésta y promueva en los diferentes espacios académicos la implementación de estrategias pedagógicas que jueguen un papel relevante en el cambio de actitudes tanto cognitivas como sociales de la población estudiantil, y por consiguiente que los universitarios se conviertan en agentes de cambio, con el fin de incidir específicamente en este caso en la disminución de las conductas sedentarias.

Entre las limitaciones identificadas en el presente estudio, se pueden mencionar principalmente la dificultad que tuvieron algunos alumnos para realizar algunas de las actividades y el tiempo que debían invertir para poder finalizarlas; situaciones que se presentaron al inicio del desarrollo del ABP, y puesto que, en la mayoría de los casos, era la primera vez que tenían contacto con este método.

Por lo anterior, se recomienda realizar una buena planificación de las actividades, con el fin de optimizar el tiempo que los alumnos les dediquen y tomar en consideración que muchas veces se les solicita a alumnos que realicen o ejecuten actividades que se asume a priori que deben saber hacer, pero que puede ser que no se les haya enseñando anteriormente.

Con el estudio se concluye que los resultados obtenidos en el diagnóstico aportaron información sobre el nivel de sedentarismo de una muestra de estudiantes de la Universidad de Costa Rica, así como el cambio en la conducta sedentaria por medio del aumento significativo en la práctica de actividad física o ejercicio realizado durante la semana, con posterioridad a la implementación del método ABP durante un curso de actividad deportiva, en el cual el método tuvo el mismo efecto positivo tanto en hombres como en mujeres. 


\section{REFERENCIAS}

1. Varela M, Duarte C, Salazar I, Lema L, Tamayo J. Actividad física y sedentarismo en jóvenes universitarios de Colombia: prácticas, motivos y recursos para realizarlas. Colomb Med. 2011; 42 (3):260-277.

2. Bolaños D. El deporte y su acción social. Kinesis. 2005; 43:31-36.

3. Hillman C, Erickson K, Kramer A. Be smart, exercise your heart: exercise effects on brain and cognition. Nat Rev Neurol. 2008; 9:58-65.

4. Rojas M. Aspectos epidemiológicos y psicológicos de la actividad y la inactividad física. En: Flórez L, Botero M y Moreno B. Psicología de la salud. Temas actuales de investigación en Latinoamérica. Bogotá: ALAPSA; 2005. p. 153-178.

5. Vainio H, Bianchini F. Physical activity and cancer prevention is 'no pain, no gain' passe? Eur J Cancer Prev. 2001; 10:301-302.

6. Organización Mundial de la Salud. Recomendaciones mundiales sobre actividad física para la salud. Ginebra, Suiza: OMS; 2010.

7. Matsud S. Actividad física: un pasaporte para la salud. Rev Med Clin Condes. 2012; 23(3):209-217.

8. Martínez L. Condición física y nivel de actividad física en estudiantes universitarios. Rev Teoria y Praxis Investigativa. 2008; 3(1):21-28.

9. World Health Organization. Health and behavior among young people. Copenhagen: WHO Regional Publications; 2000.

10. Norman G, Schmidt H. The psychological basis of problem-based learning: a review of the evidence. Acad Med. 1992; 67:557-565.

11. Campo J, Cano J, Herrera D, Bascones J, De Nova J, Gasco C, Bascones A. Aplicación de un sistema mixto de Enseñanza Tradicional/Aprendizaje Basado en Problemas en la asignatura de Urgencias en Odontología. Revista Complutense de Educación. 2009; 20(1):135-150.

12. Schuwirth L, Van der Vleuten C. ABC of learning and teaching in medicine: Written assessment. BMJ. 2003;326:643-645.

13. Smee S. ABC of learning and teaching in medicine: Skill based assessment. BMJ. 2003; 326: 703-706.
14. Spencer J. ABC of learning and teaching in medicine: Learning and teaching in the clinical environment. BMJ. 2003; 326:591-594.

15. Wood DF. ABC of learning and teaching in medicine: Problem based learning. BMJ. 2003; 326:328-330.

16. Duch J, Groh SE, Allen DE. The power of problem-based learning: A practical "bow to" for teaching undergraduate courses in any discipline. SterlingVirginia US. Stylus Publishing; 2001.

17. Gerzina TM, Worthington R, Byrne S, McMahon C. Student use and perceptions of different learning aids in a Problem-Based Learning (PBL) dentistry course. J Dent Educ. 2003; 67(6):641-53.

18. Walton HJ, Matthews MB. Essentials of problem-based learning. Med Educ. 1989; 23:542-558.

19. Barrows HS. Taxonomy of problem based learning methods. Med Educ. 1986, 20:48186.

20. Dolmans D, Schmidt HG. The advantages of problem based curricula. Postgrad Med J. 1996; 72:535-538.

21. Morales P, Landa V. Aprendizaje basado en problemas. Teoría. 2004; 13:145-157.

22. Schmidt HG, Dauphinee WD, Patel VL. Comparing the effects of Problem-Based Learning and Conventional Curricula in an international sample. J Med Educ. 1987; 62:305-315.

23. Romero T. Hacia una definición de Sedentarismo. Rev Chil Cardiol. 2009; 28:409-413.

24. Prieto A, Barbarroja J, Reyes E, Monserrat J, Díaz D, Villarroel M, et al. Un nuevo modelo de aprendizaje basado en problemas, el ABP $4 \times 4$, es eficaz para desarrollar competencias profesionales valiosas en asignaturas con más de 100 alumnos. Aula Abierta. 2006, 87:171-194.

25. López J, González M, Rodríguez M. Actividad física en estudiantes universitarios: prevalencia, características y tendencia. Med Int Mex. 2006; 22:189-196.

26. Lema L, Salazar I, Varela M, Díaz J, Rubio A, Botero A. Comportamiento y salud de los jóvenes universitarios: Satisfacción con el estilo de vida. Pens Psicol. 2009; 5:71-88. 
27. Elizondo J, Guillén F, Aguinaga I. Prevalencia de actividad física y su relación con variables sociodemográficas y estilos de vida en la población de 18 a 65 años de Pamplona. Rev Esp Salud Publica. 2005; 79:559-567.

28. Pavón A, Moreno J. Actitud de los universitarios ante la práctica físico-deportiva: Diferencias por géneros. Rev Psicol Deport. 2008; 17:7-23.

29. Yang X, Telama R, Leskinen E, Mansikkaniemi K, Viikari J, Raitakari T. Testing a model of physical activity and obesity tracking from youth to adulthood: The cardiovascular risk in young finns study. Int J Obes. 2007; 31:521-527.

30. Gámez R. Intervenciones efectivas en promoción de la actividad física. Kinesis. 2005; 42:58-61.

31. Ang E, Gómez F. Potential therapeutic effects of exercise to the brain. Curr Med Chem. 2007; 14:2564-71.

32. Valois R, Zullig K, Huebner E, Drane J. Physical activity behaviors and perceived life satisfaction among public highschool adolescents. J Sch Health. 2004; 74(2):59-65.
33. Hillman C, Erickson K, Kramer A. Be smart, exercise your heart: Exercise effects on brain and cognition. Nat Rev Neurol. 2008; 9:58-65.

34. Stevens T, To Y, Stevenson S, Lochbaum M. The importance of physical activity and physical education in the prediction of academic achievement. J Sport Behav. 2008; 31:368-88.

35. González M, Castro A. Impacto del ABP en el desarrollo de la habilidad para formular preguntas de aprendizaje en estudiantes universitarios. REDU. 2011; 9(1):57-66.

36. Gómez E, Rivas I, Mercado F, Barjola P. Aprendizaje Basado en Problemas (ABP) interdisciplinar en Ciencias de la Salud: una herramienta útil para el desarrollo de competencias profesionales. REDU. 2009; 7(4):1-19.

37. Molina J, Ortiz A, González M, Antón M. Aprendizaje basado en problemas. Una alternativa al método tradicional. REDU. 2003; 3(2):79-85. 